\title{
EVALUATION OF CT SCAN FINDINGS IN PATIENTS PRESENTING WITH HEADACHE
}

\author{
P Nepal $^{1 *}$, A Shrestha ${ }^{2}$, Ghimire $^{3}$ \\ ${ }^{1}$ Department of Radiodiagnosis and Imaging, Chitwan Medical College, Bharatpur-10, Chitwan, Nepal. \\ ${ }^{2}$ Department of Neurosurgery, Chitwan Medical College, Bharatpur-10, Chitwan, Nepal. \\ ${ }^{3}$ Department of Pedodontics and Preventive Dentistry, Chitwan Medical College, Bharatpur-10, Chitwan, Nepal. \\ *Correspondence to : Dr Pankaj Nepal, Department of Radiodiagnosis and Imaging, Chitwan Medical College, Bharatpur-10, Chitwan, Nepal. \\ Email: pankaj-123@live.com
}

\begin{abstract}
Headache is one of the most common medical complaint however, the secondary causes of headache are unusual. Neuroimaging in headache is both expensive and has a low yield. Most patients with intracranial pathology have alarming clinical features raising " red flag" signs. Thus appropriate selection of patients with headache for neuroimaging to look for secondary cause is important. Red flags act as screening tool to help in identifying those who get benefit from neuroimaging. These steps not only increase yield, but also limit cost as well as burden in health care system. The aim of our study was to evaluate all the CT scan findings in patients presenting with headache in Chitwan Medical College who underwent CT scan of head from February 2013 to September 2013 for period of seven months. Prospective observational study was performed in 256 patients, who underwent CT scan of head in this duration. Out of 256 patients, only 26 patients (10.1\%) showed some form of brain parenchymal pathology. Other associated findings were sinusitis in $28(11 \%)$, bone related in $10(3.9 \%)$, and mastoiditis in $6(2.3 \%)$ patients. The most prevalent pathology found was sinusitis, which was located in the maxillary sinus. The findings were significant in headache associated with other neurological findings, 16 out of 24 patients $(67 \%)$ as compared to chronic headache in which only 10 cases out of $232(4.3 \%)$ patients were positive $(\mathrm{p}<0.05)$. Cases referred from neurosurgery department and emergency yielded more positive results as compared to case referred from other outpatient departments.
\end{abstract}

Key Words: CT Scan, Headache, Neurological Symptoms.

\section{INTRODUCTION}

Headache is one of the most common medical complaints. However only about $10 \%$ of patients with headache were due to secondary causes. ${ }^{1}$ As the potential etiologies, can be life threatening and affect neurological function, headache cause understandable concern on the part of the patient and health care provider. $^{2}$ Some serious brain disorders present with secondary headache, where the headache is caused by the disease. For example, a brain tumor is secondary cause of headache and is best diagnosed by early brain imaging, which is essential for optimal management of secondary headache disorders. However, brain tumors, account for less than $0.1 \%$ of the lifetime prevalence of headache. ${ }^{3}$

Discriminating between primary and secondary headache is the problem. Since by definition, primary headache does not need brain imaging because no underlying disease process exists. Primary headache, which include migraine, tension-type headache and cluster headache are benign. These headaches are usually recurrent and have no organic disease as their cause. Secondary headaches are caused by underlying organic diseases ranging from benign condition as sinusitis to life threatening causes like subarachnoid hemorrhage. ${ }^{4}$ In clinical practice, it is generally accepted that the so called red flags of headache should lead to a search for secondary headache. ${ }^{5}$

These include:

1. Change in the pattern of headache,

2. New onset of headache in people older than 50,

3. Onset of seizures or headache associated with systemic illness or personality change,

4. Headache with symptoms suggestive of raised intracranial pressure, such as new onset headache in the early morning; or headache that is worsening with coughing, sneezing, or straining should each be viewed with concern

A detailed and dedicated headache history and thorough neurological and general physical examination should help in deciding which patients with headache need CT Scan of brain, to find out the underlying brain pathology. At times despite the absence of red flags, a CT scan of brain is requested to allay the anxiety of the patient and relatives. But this raises the issue of radiation dose to the patient especially in young children. Computed tomography accounts for $40 \%$ of medical diagnostic 
radiation but represents only $4 \%$ of radiology examinations. ${ }^{6}$ The use of computed tomography has to be balanced against the radiation dose. Computed tomography is a highly useful tool for solving problems, but it should never be allowed to replace proper history taking and clinical examinations.

\section{RESULTS}

Collected data was analyzed using SPSS programme. For the continuous data, mean and standard deviation were considered while for the categorical data proportion and percentage were used. Observational study was performed in all the 256 patients who underwent CT scan of head from February 2013 to September 2013. In this duration, 256 patients underwent CT scan of head. The proforma was prepared and patients were grouped into two groups. The group I represented the cases with headache and any form of neurological deficit as indicated in red flag signs. The Group II represented the cases of chronic headaches not associated with any red flag signs. The association between group I and II or type of headache and positive findings were established using chi square test. 25 out of all the cases underwent contrast administration as per the indications.

The age group ranged from 9 years to 87 years with the mean of 35 years. Group I included 24 cases whereas 232 cases were included in Group II. $73 \%$ of the CT scans were absolutely normal. Out of 256 patients only $26(10.1 \%)$ showed any form of brain parenchymal pathology.

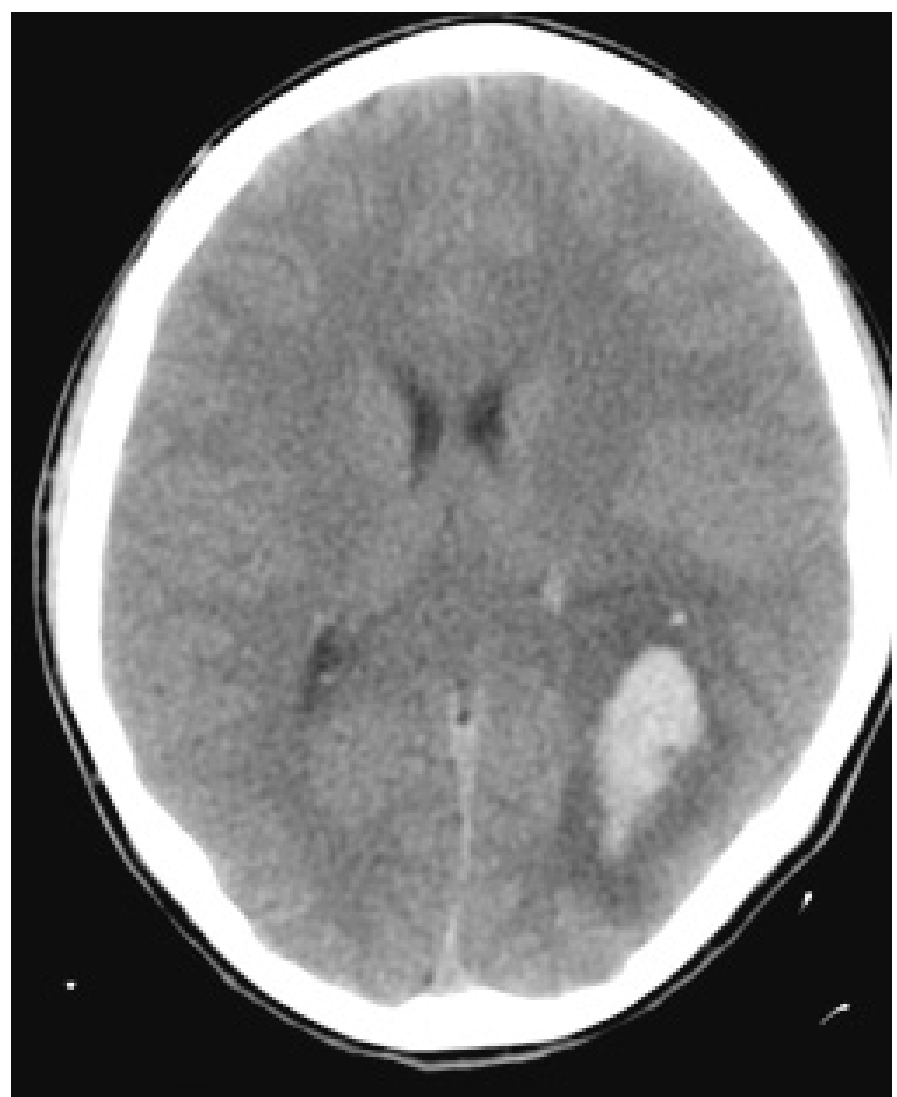

Figure 1: Brain parenchymal cause of headache showing hypertensive bleed in left parietooccipital region.

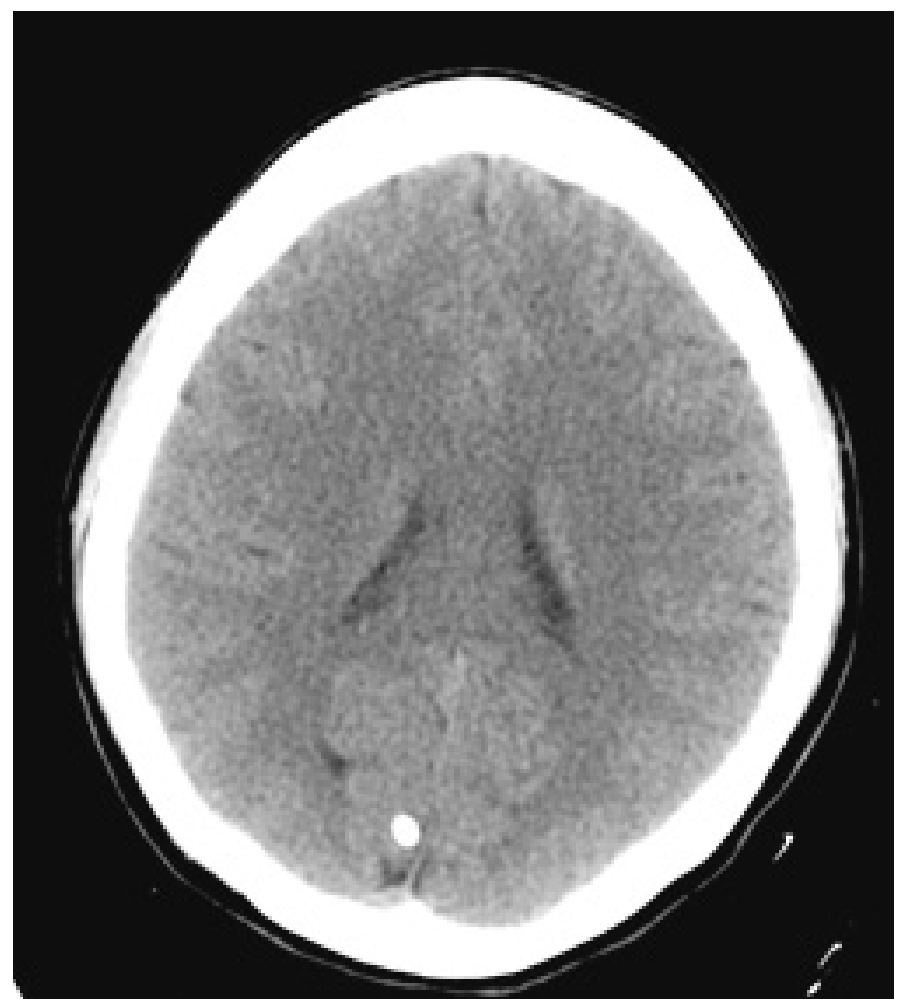

Figure 2: Indeterminate finding;calcified granuloma in right occipital lobe in chronic headache patient.

Other associated findings were sinusitis in $28(11 \%)$, bone related in $10(3.9 \%)$, and mastoiditis in $6(2.3 \%)$ patients.

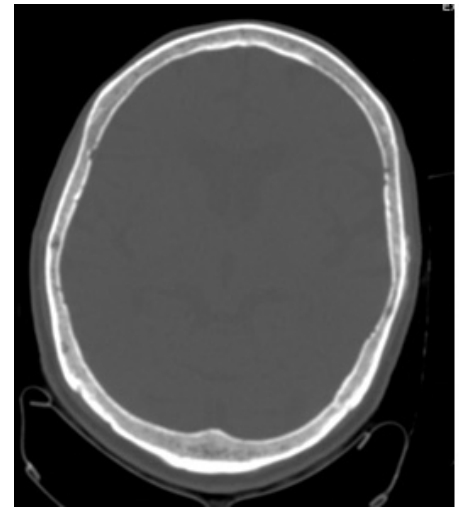

a

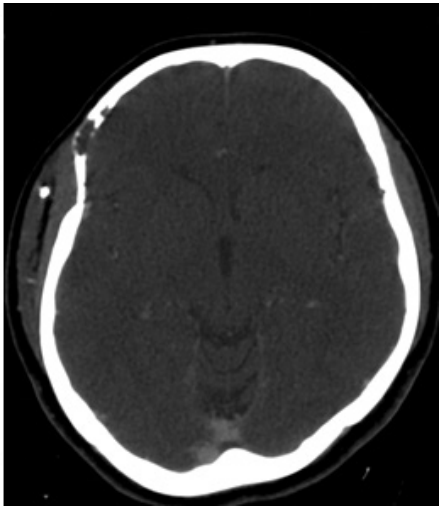

b
Figure 3: a, b Non brain parenchymal causes of headache; multiple lytic lesions in skull in multiple myeloma (left) and parotid mass with bone destruction (right).

Out of the 26 brain parenchyma pathology, 10 were cases of calcified granulomas, 5 ring enhancing lesions, 3 cases of presumptive brain tumor, 3 cases of parenchymal bleed, 2 cases of presumptive demyelination, 2 case of subarachnoid hemorrhage and 1 case of aneurysm. In overall the most prevalent pathology found was sinusitis which was located in the maxillary sinus. 


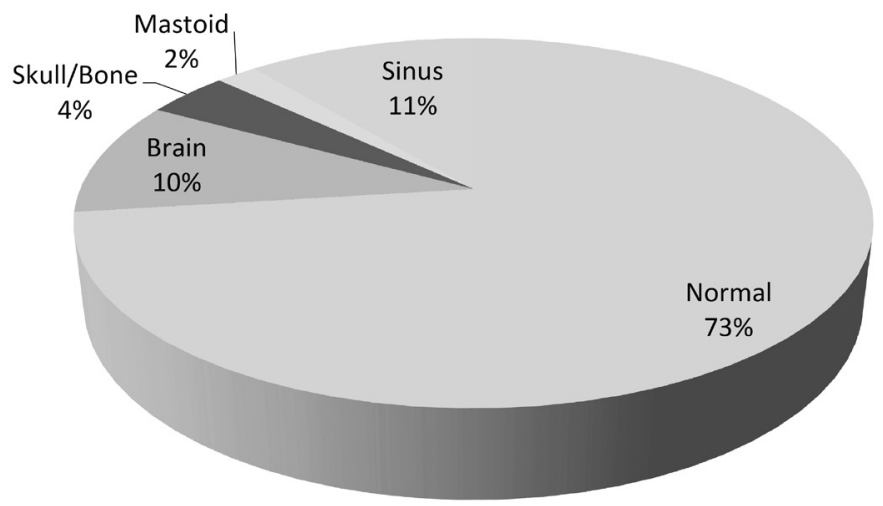

Chart I: Abnormalities detected in CT scans of all patients. However only $10 \%$ of brain abnormalities were detected.

The findings were significant in headache associated with other neurological findings, 16 out of 24(67\%) in group I as compared to chronic headache (Group II) in which 10 cases out of 232( $4.3 \%)$ were positive $(\mathrm{p}<0.05)$.

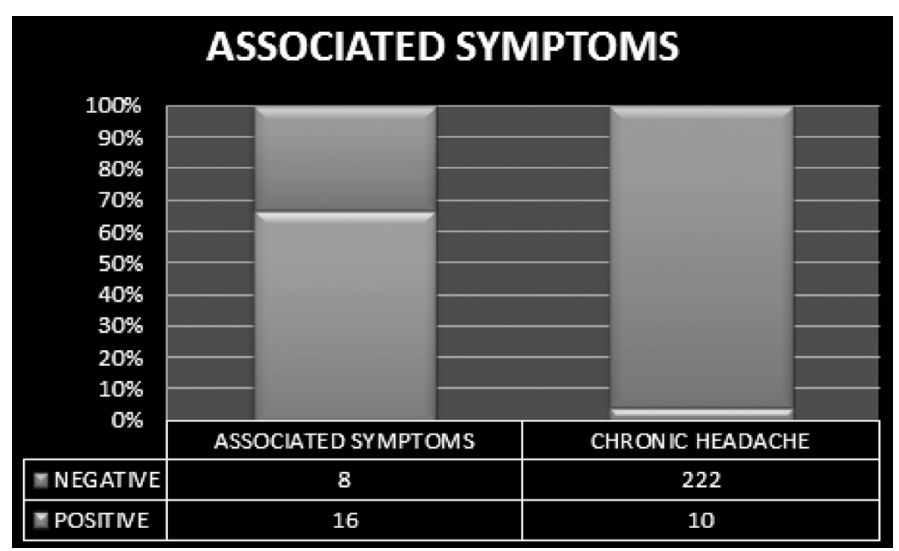

Table 1: Group I on left and Group II on right. The patients with "red flag signs" show significant findings compared to chronic headache cases

Cases referred from neurosurgery department and emergency yielded more positive results compared to reffered from other outpatient departments.

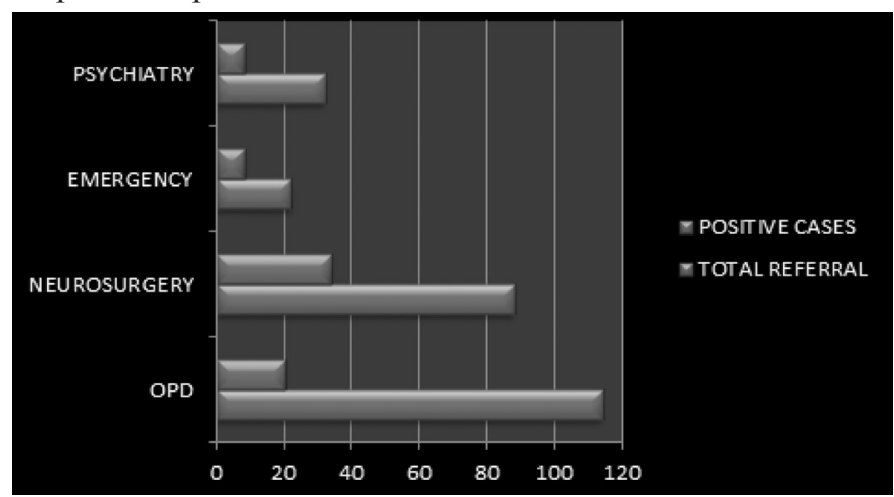

Table 2: Case referral from various departments. Note cases referred from emergency and neurosurgery were significantly positive

\section{DISCUSSION}

The International Headache Society classifies headache into primary (without any organic cause) or secondary (with an established cause). Primary headache includes migraine, cluster type headaches. With these types of headaches no imaging modality has proved to be necessary.

Various studies have been conducted to asses the utility of CT scanning in patients of headache. In one such study conducted at Saidu Medical College \& Teaching Hospital in Swat, Pakistan, it was found that $69 \%$ of patients presenting with sudden thunderclap headache had no abnormality in CT scans of brain. ${ }^{7}$

A study was conducted at Samsun, Turkey, to investigate the frequency of intracranial lesions detected by CT scanning amongst adult patients who had clinical warning criteria (CWC) for secondary neurological headaches and to determine the importance of CWC in predicting a possible lesion on CT scan. ${ }^{8}$ The CWC included:

1. Increase in the intensity and frequency of headache,

2. Abrupt onset of headache,

3. Persistence of headache despite analgesics,

4. Alteration of the characteristics of headache and

5. Presence of focal neurological symptoms or findings.

Out of all the patients, $35.7 \%$ had a neurological cause identified by CT scan and $64.3 \%$ had a normal CT scan. In our study $67 \%$ of the patients with red flag signs had an abnormality on CT scan of brain compared to only $4.3 \%$ of the patients without associated neurological findings.

A study of pediatric patients was done at George Washington University School of Medicine, Washington, DC, to determine whether CT scans led to better acute care of young children with headache presenting to the emergency department. It was found that for young children presenting with headache but normal neurological examination and non-worrying history, CT scans seldom lead to diagnosis or contribute to immediate management. ${ }^{9}$ The use of CT scanning in young children should be done very carefully to avoid the hazardous exposure to radiation at a young age.

Previous studies have demonstrated that CT is of extremely low yield in patients who undergo imaging for chronic headache without neurologic abnormality. ${ }^{11}$ Our study show $27 \%$ abnormal scans out of all the cases who underwent CT scans for headache. However pure brain parenchymal pathology was seen only in $10 \%$ of cases. In these cases too it was a matter of confusion to perceive if calcified or inactive lesions contribute to headache. Also the associated symptoms may not represent true cause of headache, for example sinusitis may be incidental finding in case of headache. In addition it may add confusion and anxiety in normal cases.

MR imaging is even an unrewarding technique in the evaluation of patients with chronic or recurrent headache and normal neurologic findings. Neither contrast enhancement nor repeated MR imaging contributed to diagnosis in the evaluation of Chronic or Recurrent Headache. A study was conducted at Gunma University Hospital, Gunma, Japan, to evaluate the ability of magnetic resonance imaging (MRI) to depict an abnormality in patients with chronic or recurrent headache without neurologic abnormality. ${ }^{10}$ Out of a total of 306 patients, $169(55.2 \%)$ had no abnormality, $135(44.1 \%$ ) had a minor abnormality while only two 
$(0.7 \%)$ had a clinically important abnormality at MR imaging.

Although the majority of patients who present with chronic or recurrent headache have no neurologic abnormality, many patients undergo evaluation with computed tomography (CT) and, more recently, magnetic resonance (MR) imaging to exclude important abnormalities. ${ }^{12,13,14}$ Thus our endeavour should be to reduce the radiation burden, as CT scan involves considerable radiation. Our study group showed maximum patients in reproductive age group. Unnecessary CT scans lead to the radiation and its side effect in this vulnerable age group.

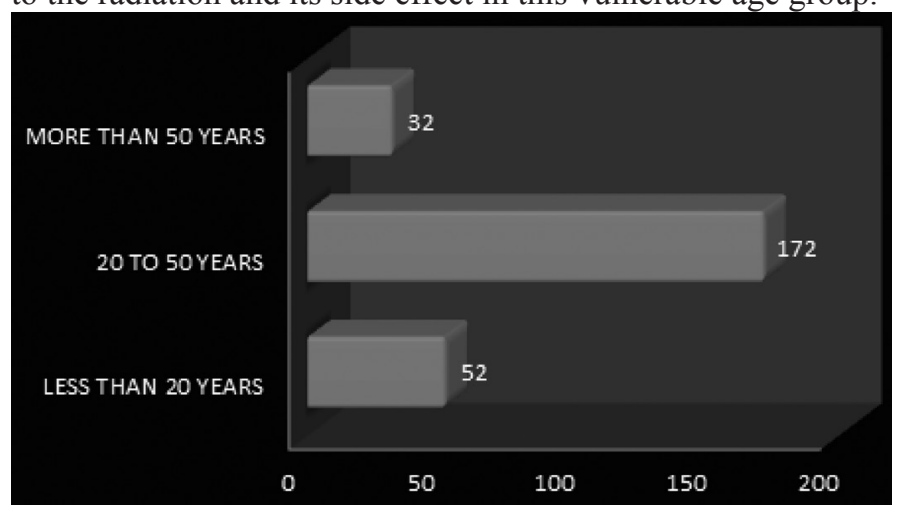

Table IV: Age distribution. Maximum cases belong to reproductive age group

Thus the Yield of CT scans in group I was statically significant to the Group II. Use of CT scans must be ethical and selective. It is mandatory to image the patients with red flag signs or warning symptoms as discussed above. The associated symptoms like sinusitis may be incidental findings and falsely increasing the yield of CT.

\section{CONCLUSION}

CT scan of brain is rarely of help in the diagnosis of headache if it is not associated with other symptoms and or signs of an intracranial pathology. When to scan a patient for headache is a dilemma faced by physicians in their daily practice. Patients with red flags or clinical warning criteria of secondary headache probably must undergo a CT scan of brain. In the absence of these, the only reason for doing a CT scan seems to be reassuring the patients and their loved ones.

\section{REFRENCES}

1. Perkins AT, Ondo W. When to worry about headache; Head pain as a clue to intracranial disease. Postgrad Med 1995;98:197-208.

2. Bartleson JD. When and how to investigate the patient with headache. Semin Neurol 2006; 26;2: 163-70.

3. Goadsby PJ. To scan or not to scan in headache. Editorial. BMJ 2004; 329: 469-70.

4. Clinch CR. Evaluation of acute headaches in adults. Am Fam Physician 2001; 15; 63;4: 685-92.

5. Frishberg BM. The utility of neuroimaging in the evaluation of headache in patients with normal neurologic examinations.Neurology1994; 44:1191-1197.

6. Garvey CJ, Hanlon R. Computed tomography in clinical practice. Clinical review. BMJ 2002; 324: 1077-80.

7. Ahmad A, Khan P, Ahmad K, Syed A. Diagnostic outcome of patients presenting with severe thunderclap headache at
Saidu teaching hospital. Pak J Med Sci 2008; 24: 3: 575-80.

8. Aygun D, Bildik F. Clinical warning criteria in evaluation by computed tomography of the secondary neurological headaches in adults. Eur J Neurol 2003; 10;4: 437-42.

9. Lateef TM, Grewal M, McClintock W, Chamberlain J, Kaulas H, Nelson KB. Headache in young children in the emergency department: use of computed tomography. Pediatrics 2009; 124;1: 12-7.

10. Tsushima Y, Endo K. MR Imaging in the evaluation of chronic or recurrent headache Radiology 2005; 235: 575-9.

11. Dumas MD, Pexman JH, Kreeft JH. Computed tomography evaluation of patients with chronic headache. Can Med Assoc J.1995; 152:158.

12. Report of the Quality Standards Subcommittee of the American Academy of Neurology.Neurology1994; 44:13531354.

13. Steiner TJ, Fontebasso M. Headache. Clinical review. BMJ 2002; 325: 881-886.

14. Clinch CR. Evaluation of acute headaches in adults. Am Fam Physician 2001; 63 : 685-92. 